\title{
Arpin downregulation in breast cancer is associated with poor prognosis
}

\author{
Maria E Lomakina ${ }^{1}$, François Lallemand ${ }^{2}$, Sophie Vacher ${ }^{2}$, Nicolas Molinie ${ }^{3}$, Irene Dang ${ }^{3}$, Wulfran Cacheux ${ }^{2,4}$, \\ Tamara A Chipysheva ${ }^{1}$, Valeria D Ermilova ${ }^{5}$, Leanne de Koning ${ }^{6}$, Thierry Dubois ${ }^{6}$, Ivan Bièche ${ }^{2}$, \\ Antonina Y Alexandrova ${ }^{1}$ and Alexis Gautreau ${ }^{\star, 3}$ \\ ${ }^{1}$ Institute of Carcinogenesis, N.N. Blokhin Cancer Research Center, Kashirskoe shosse 24, Moscow 115478, Russian Federation; \\ ${ }^{2}$ Pharmacogenomics Unit, Department of Genetics, Institut Curie, 26 rue d'Ulm, 75005 Paris, France; ${ }^{3}$ Ecole Polytechnique, CNRS \\ UMR7654, Route de Saclay, 91128 Palaiseau, France; ${ }^{4}$ Department of Medical Oncology, Institut Curie, 26 rue d'Ulm, 75005 Paris, \\ France; ${ }^{5}$ Department of Pathology, N.N. Blokhin Cancer Research Center, Moscow 115478, Russia and ${ }^{6}$ Department of \\ Translational Research, Institut Curie, PSL Research University, 26 rue d'Ulm, 75005 Paris, France
}

Background: The Arp2/3 complex is required for cell migration and invasion. The Arp $2 / 3$ complex and its activators, such as the WAVE complex, are deregulated in diverse cancers. Here we investigate the expression of Arpin, the Arp2/3 inhibitory protein that antagonises the WAVE complex.

Methods: We used qRT-PCR and reverse phase protein arrays in a patient cohort with known clinical parameters and outcome, immunofluorescence in breast biopsy cryosections and breast cancer cell lines.

Results: Arpin was downregulated at the mRNA and protein levels in mammary carcinoma cells. Arpin mRNA downregulation was associated with poor metastasis-free survival (MFS) on univariate analysis $(P=0.022)$. High expression of the NCKAP1 gene that encodes a WAVE complex subunit was also associated with poor MFS on univariate analysis $(P=0.0037)$ and was mutually exclusive with Arpin low. Arpin low or NCKAP1 high was an independent prognosis factor on multivariate analysis $(P=0.0012)$ and was strongly associated with poor MFS $(P=0.000064)$.

Conclusions: Loss of the Arp2/3 inhibitory protein Arpin produces a similar poor outcome in breast cancer as high expression of the NCKAP1 subunit of the Arp2/3 activatory WAVE complex.

The actin cytoskeleton is critical for a variety of cell functions including control of shape, cell migration and intracellular traffic. Branched actin networks in particular were found to generate a pushing force that drives membrane protrusion during cell migration (Insall and Machesky, 2009). This pushing force also promotes membrane constriction and hence scission of transport intermediates in intracellular traffic. The Arp2/3 complex is the sole machinery that generates branched actin networks. This machinery is activated at different locations of the cell by activators, called Nucleation Promoting Factors (NPFs; Rotty et al, 2013).
In untransformed cells, different NPFs have distinct functions. For example, WAVE proteins are consistently found at the leading edge and generate the membrane protrusions that allow cell migration, whereas N-WASP contributes to endocytosis by performing scission of clathrin-coated pits (Rotty et al, 2013). Such clear distinctions are blurred in tumour cells, where Arp2/3 regulation is altered. For example, invasion of tumour cells depends on N-WASP and on the formation of novel protrusive structures that degrade the extracellular matrix, called invadopodia (Yamaguchi et al, 2005). The WAVE function in normal cell migration is thus replaced by N-WASP-dependent dissemination

*Correspondence: Professor A Gautreau; E-mail: alexis.gautreau@polytechnique.edu

revised 8 December 2016; accepted 6 January 2016; published online 11 February 2016

(c) 2016 Cancer Research UK. All rights reserved 0007-0920/16 
of tumour cells and formation of metastasis (Tang et al, 2013). Loss of WAVE activity even stimulates 3D invasion of carcinoma cells by activating N-WASP (Tang et al, 2013).

There is a consensus to recognise that the Arp2/3 complex is required for tumour cell migration and invasion (Liu et al, 2013; Rauhala et al, 2013; Frugtniet et al, 2015). In patients, Arp $2 / 3$ is overexpressed and this overexpression is associated with poor prognosis in various cancers including breast cancer (Otsubo et al, 2004; Semba et al, 2006; Iwaya et al, 2007a, b; Laurila et al, 2009). There is evidence for both N-WASP- and WAVE-mediated dissemination of tumour cells in patients. WAVE proteins are regulated within a multisubunit complex (Derivery and Gautreau, 2010) and several subunits of the complex have been described as tumour suppressors (Silva et al, 2009; Xiong et al, 2012; Sowalsky et al, 2015). However, reported cases of Arp $2 / 3$ overexpression were associated with WAVE overexpression (Semba et al, 2006; Iwaya et al, 2007a, b). Studies reporting overexpression of WAVE family members in various cancers are actually more frequent than the ones reporting N-WASP overexpression (Kurisu and Takenawa, 2010). In breast cancer, overexpression of the WAVE3 subunit appears associated with invasiveness and poor prognosis (Sossey-Alaoui et al, 2007; Kulkarni et al, 2012). Consistent with a mutual exclusion between WAVE and N-WASP, N-WASP downregulation was reported in breast cancer to be associated with invasiveness and poor prognosis (Martin et al, 2007).

Recently, inhibitory proteins that prevent Arp $2 / 3$ activation by NPFs were identified. Interestingly, these proteins are differentially localised in the cell, like NPFs. PICK1 is localised at clathrin-coated pits of the plasma membrane, where it can be envisioned to antagonise N-WASP (Rocca et al, 2008). Gadkin localises at the surface of endosomes, where it sequesters the Arp2/3 complex in order to prevent its use in membrane protrusion and cell migration (Maritzen et al, 2012). Most recently, we reported Arpin, an Arp2/3 inhibitory protein that localises at the leading edge of migrating cells. Arpin antagonises the WAVE complex (Dang et al, 2013). Strikingly, the small GTPase Rac, which activates the WAVE complex, also activates Arpin. Thereby, Arpin controls protrusion lifetime and migration persistence (Krause and Gautreau, 2014). To further characterise how Arp $2 / 3$ deregulation contributes to breast cancer, we examined the expression of these three Arp $2 / 3$ inhibitory proteins in patient biopsies.

\section{MATERIALS AND METHODS}

Patients and samples. Samples of 454 unilateral invasive primary breast tumours excised from women managed at Institut CurieHôpital René Huguenin (Saint-Cloud, France) from 1978 to 2008 have been analysed. All patients who entered our institution before 2007 were informed that their tumour samples might be used for scientific purposes and had the opportunity to decline. Since 2007, patients entering our institution have given their approval also by signed informed consent. This study was approved by the local ethics committee (Breast Group of René Huguenin Hospital). All patients had primary unilateral non-metastatic breast carcinoma for which complete clinical, histologic and biological data were available. Before surgery, no radiotherapy or chemotherapy were performed. During a median follow-up of 8.9 years (range 6 months to 29 years), 167 patients metastasised. Ten specimens of adjacent normal breast tissue from breast cancer patients or normal breast tissue from women undergoing cosmetic breast surgery were used as sources of normal RNA.

For immunofluorescence, samples were obtained from patients who had undergone a mastectomy without preliminary therapy, at the N.N. Blokhin Russian Cancer Research Center. The Institutional Review Board of N.N. Blokhin Russian Cancer Research
Center approved the project and all patients, who were involved in the study, gave written informed consents that their samples could be used for investigational purposes. Data were analysed anonymously. All potential participants who declined to participate or otherwise did not participate were eligible for treatment (if applicable) and were not disadvantaged in any other way by not participating in the study.

Untreated human samples from colorectal adenomas $(n=43)$, primary colorectal carcinomas $(n=67)$, liver metastasis $(n=27)$ and matched normal adjacent colorectal tissues $(n=103)$ were collected and analysed at the Institut Curie.

The origin of breast cell lines and conditions for their culture were described previously (Maire et al, 2013).

qRT-PCR. Total RNA was extracted from normal and malignant breast samples using the acid-phenol guanidium protocol. RNA quality was determined by electrophoresis through agarose gels. The gene-specific primers were chosen with the assistance of the Oligo 6.0 programme (National Biosciences, Colorado Springs, CO, USA). $\mathrm{dbEST}$ and nr databases were scanned to confirm the specificity of the nucleotide sequences chosen for the primers and the absence of single-nucleotide polymorphisms. To avoid amplification of potential contaminating genomic DNA, one of the two primers was placed at the junction between two exons. Agarose gel electrophoresis was used to verify the specificity of PCR amplicons. The nucleotide sequences of the primers used were as follows: ARPIN-U (5'-CTTCCTCATGTC GTCCTACAAGGTG-3'), ARPIN-L (5'-CTGTCAGCGCGAGCAGC TCT-3'), PICK1-U2 (5'-GGACACTCGCCTCACCATCAAGA-3'), PICK1-L2 (5'-GCTCGCCTAGGGCAATGCAG-3'), AP1AR-U2 (5'-C GGAGGATCCAAGTATTTTAGAACAT-3'), AP1AR-L2 (5'-CTGGG CTCTCCCCTTCATCA-3'), NCKAP1-U2 (5' -AGTGTACCCTTAGTGACCAGTTGCT-3'), NCKAP1-L2 (5'-TCAGGTTCCCCTTTCTTAC CAGT- $\left.3^{\prime}\right)$, TBP-U (5'-TGCACAGGAGCCAAGAGTGAA- $\left.3^{\prime}\right)$ and TBP-L (5'-CACATCACAGCTCCCCACCA- $\left.3^{\prime}\right)$. The conditions of cDNA synthesis and PCR were previously described (Bieche et al, 2001). Each sample was normalised on the basis of its TBP content. Results, expressed as $\mathrm{N}$-fold differences in specific gene expression relative to the TBP gene were determined as $N=2^{\Delta \text { Ctsample }}$, where the $\Delta \mathrm{Ct}$ value of the sample was determined by subtracting the average $\mathrm{Ct}$ value of the specific gene from the average $\mathrm{Ct}$ value of the TBP gene. The $N$ values of the breast or colon tumour samples were subsequently normalised such that the median for the normal tissues was 1 .

Reverse phase protein array (RPPA). RPPA was performed exactly as previously described (Rondeau et al, 2015).

Immunofluorescence of biopsy cryosections. Biopsies were frozen in liquid nitrogen and serially sectioned. Sections were fixed with acetone-methanol and stained with Arpin rabbit polyclonal antibodies, keratin 8 (clone $\mathrm{H} 1, \mathrm{IgG} 1$ ) and keratin 17 (clone E3, IgG2b) mouse mAbs followed by fluorescent anti-rabbit and isotype-specific anti-mouse antibodies (Southern Biotechnology, Birmingham, AL, USA; Guelstein et al, 1988). Specific fluorescence intensity of Arpin, $I$, was calculated from the intensity in specific regions, $S$, and the one in a surrounding region of same size, $C$, according to the formula $I=(S-C) / C$. An average per biopsy was calculated from 5 to 10 micrographs using 20 to 30 regions per micrograph.

Statistical analysis. The distributions of mRNA levels were characterised by their median values and ranges. Relationships between mRNA levels of the different target genes, and between mRNA levels and clinical parameters, were identified using nonparametric tests, namely the $\chi^{2}$ test (relation between two qualitative parameters), the Mann-Whitney $U$-test (relation between one qualitative parameter and one quantitative parameter) and the Spearman rank correlation test (relation between two quantitative parameters). Differences were considered significant at confidence levels greater than 95\% $(P<0.05)$. 
Metastasis-free survival was determined as the interval between initial diagnosis and detection of the first metastasis. Survival distributions were estimated by the Kaplan-Meier method, and the significance of differences between survival rates were ascertained with the log-rank test. Cox's proportional hazard regression model was used to assess prognostic significance and the results are presented as hazard ratio and $95 \%$ confidence intervals.

Western blot of cell line extracts. Cells were extracted with SDS-PAGE loading buffer. Protein concentration in extracts was quantified using the BCA kit (Life Technologies, Courtaboeuf, France). $10 \mu \mathrm{g}$ of total proteins were loaded on NuPAGE $4-12 \%$ Bis-Tris gels (Life Technologies) run in MES buffer. Proteins were transferred using iBlot system (Life Technologies) and developed using HRP-coupled antibodies and the Supersignal kit (Life Technologies) and a LAS-3000 imager (Fujifilm, Düsseldorf, Germany). Arpin protein level was quantified on western blot membranes selected at equal exposure time. Band intensities were measured on ImageJ (Bethesda, MD, USA) by quantifying the peak area of each band corrected by background subtraction.

Assays utilising MDA-MB-231 clones depleted or not of Arpin. MDA-MB-231 clones depleted or not of Arpin were previously obtained (Dang et al, 2013). They were grown in RPMI containing 10\% FBS. Growth curves of these clones were obtained using the CellTiter 96 AQueous One Solution Cell Proliferation Assay kit (Promega, Charbonnieres, France) according to the manufacturer's instructions. Wound healing assays were performed using two chambers where cells were grown to confluence (culture-insert, Ibidi). After removal of the inserts, wound healing was monitored live for $10 \mathrm{~h}$ by phase contrast using the Plan-Apochromat $10 \times / 0.80$ air objective of an axioobserver Z1 microscope (Zeiss, Marly le Roi, France). Soft agar growth was performed by seeding 10000 cells in $0.35 \%$ agar noble (Sigma, Saint-Quentin Fallavier, France) in 12-well plates in a volume of $500 \mu \mathrm{l}$. After 21 days in culture, colonies were imaged by phase contrast. Transformation foci were obtained by seeding 500 cells in wells of 12-well dishes, incubated for 14 days and stained by crystal violet. Quantitation was obtained by dissolving the stain in $10 \%$ acetic acid and by measuring the absorbance at $570 \mathrm{~nm}$.

\section{RESULTS}

Arpin downregulation in breast cancer is associated with poor prognosis. To examine the expression of Arp2/3 inhibitory proteins in breast cancer, we used a retrospective cohort of patients, which was started more than 30 years ago. This allowed us to examine if variations in the mRNA expression of Arp2/3 inhibitory proteins had a value for patient prognosis. In the cohort of 454 patients, we examined the expression of Arpin (also known as C15ORF38), PICK1 and Gadkin (also known as $A P 1 A R$ ) using quantitative RT-PCR. We defined overand underexpression as at least three-fold higher or lower expression than the median expression observed in healthy breast tissue. Using this definition, more than $97 \%$ of tumours displayed normal expression of PICK1. In contrast, $8.5 \%$ of patients overexpressed Gadkin and $7.3 \%$ downregulated Arpin expression, with the same criteria (Figure 1A).

Arpin downregulation was weakly associated with a large tumour size and a high Scarff Bloom Richardson (SBR) grade (Table 1 and Supplementary Figure 1). Gadkin overexpression was weakly associated with old age of patients, intermediate SBR grade, with positive ER status, and hence, the molecular subtype of breast cancers (Supplementary Table 1). As for PICK1, the quantity of its mRNA was weakly associated with the molecular subtype of breast carcinomas, and in particular with the ERBB2 status (Supplementary Table 2).

Most importantly, Arpin downregulation was associated with reduced MFS in a statistically significant manner (Figure 1B; $P=0.022$ ). Indeed, in patients displaying reduced Arpin expression, there was only $51.6 \%$ MFS $v$ s $75.5 \%$ for patients displaying normal Arpin expression at 5 years (60 months) and $44.2 \%$ vs

\begin{tabular}{|l|c|c|c|}
\hline Aumber of tumours $(\%)$ & Arpin $^{*}$ & PICK1 $^{\text {** }}$ & Gadkin $^{\text {** }}$ \\
\hline Under-expressing tumours $(N$ target $<0.33)$ & $33(7.3)$ & $2(0.4)$ & $5(1.1)$ \\
Normo-expressing tumours $(0.33 \leqslant N$ target $\leqslant 3)$ & $414(91.2)$ & $436(97.8)$ & $403(90.4)$ \\
Over-expressing tumours $(N$ target $>3)$ & $7(1.5)$ & $8(1.8)$ & $38(8.5)$ \\
& & & \\
\hline
\end{tabular}

B
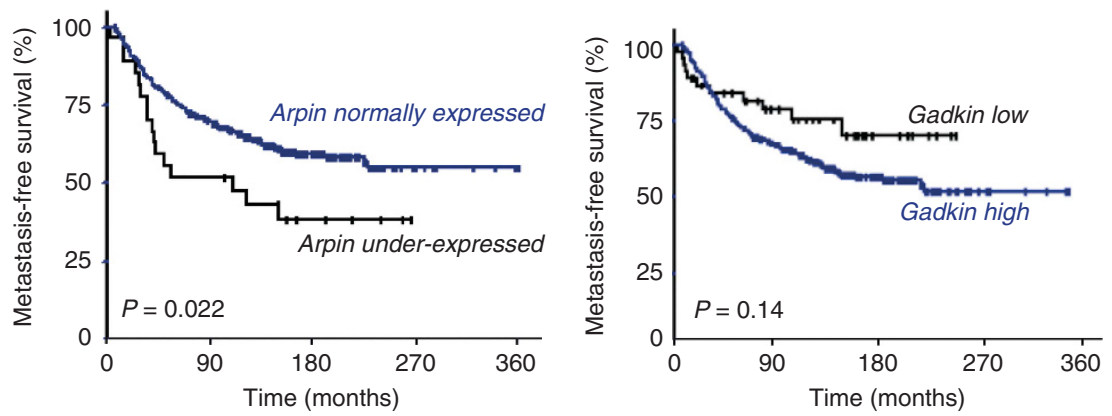

Figure 1. Arpin mRNA under-expression is associated with poor prognosis in breast cancer. (A) Expression of the three Arp2/3 inhibitors, Arpin, PICK1 and Gadkin was examined in a large series of breast tumours from patients and in 10 normal breast tissues. mRNA values were quantified using qRT-PCR. Values of breast cancer samples were normalised to the median of the 10 normal breast tissue values. *Information available for 454 tumours, **information available for 446 tumours. PICK1 expression did not significantly vary among the breast tumours. In contrast, expression of Arpin (C15ORF38) and Gadkin (AP1AR) genes varies in a significant number of tumours, Arpin being under-expressed and Gadkin overexpressed. (B) Metastasis-free survival (MFS) of patients was determined as the interval between initial diagnosis and detection of the first metastasis. Survival distributions in the different groups of gene expression were plotted using the Kaplan-Meier method, and the significance of the difference was ascertained with the log-rank test using optimal cutoffs ( 0.31 for Arpin and 0.83 for Gadkin). Whereas Gadkin expression does not significantly affect MFS $(P=0.14)$, patients harbouring tumours with reduced Arpin mRNA expression have a significant poorer prognosis compared with the others $(P=0.022)$. 
Table 1. Characteristics of breast tumours relative to Arpin mRNA

\begin{tabular}{|c|c|c|c|c|c|c|}
\hline & \multicolumn{3}{|c|}{ Prognosis status } & \multicolumn{3}{|c|}{ Arpin status } \\
\hline & $\begin{array}{c}\text { Total population } \\
(\%)\end{array}$ & $\begin{array}{c}\text { Metastases } \\
(\%)\end{array}$ & $P$-value ${ }^{a}$ & $\begin{array}{c}\text { Arpin mRNA } \\
\text { under expressed }\end{array}$ & $\begin{array}{c}\text { Arpin mRNA } \\
\text { normally } \\
\text { expressed }\end{array}$ & $P$-value ${ }^{\mathrm{b}}$ \\
\hline Total & $454(100.0)$ & 167 (36.8) & & $33(7.3)$ & 421 (92.7) & \\
\hline \multicolumn{7}{|l|}{ Age (years) } \\
\hline $\begin{array}{l}\leqslant 50 \\
>50\end{array}$ & $\begin{array}{r}97(21.4) \\
357(78.6)\end{array}$ & $\begin{array}{r}36(21.6) \\
131(78.4)\end{array}$ & 0.63 (NS) & $\begin{array}{r}9(27.3) \\
24(72.7)\end{array}$ & $\begin{array}{r}88(20.9) \\
333(79.1)\end{array}$ & 0.39 (NS) \\
\hline \multicolumn{7}{|c|}{ SBR histologic grade $e^{c, d}$} \\
\hline $\begin{array}{l}\text { I } \\
\text { II } \\
\text { III }\end{array}$ & $\begin{array}{r}58(13.0) \\
228(51.2) \\
159(35.7)\end{array}$ & $\begin{array}{c}8(4.9) \\
83(50.9) \\
72(44.2)\end{array}$ & 0.00011 & $\begin{array}{c}1(3.0) \\
13(39.4) \\
19(57.6)\end{array}$ & $\begin{array}{r}57(13.8) \\
215(52.2) \\
140(34.0) \\
\end{array}$ & 0.014 \\
\hline \multicolumn{7}{|c|}{ Lymph node status $^{\mathrm{e}}$} \\
\hline $\begin{array}{l}0 \\
1-3 \\
>3\end{array}$ & $\begin{array}{r}119(26.3) \\
237(52.3) \\
97(21.4)\end{array}$ & $\begin{array}{l}35(21.0) \\
77(46.1) \\
55(32.9)\end{array}$ & 0.0000027 & $\begin{aligned} 10 & (30.3) \\
16 & (48.5) \\
7 & (21.2)\end{aligned}$ & $\begin{array}{r}109(26.0) \\
221(52.6) \\
90(21.4)\end{array}$ & 0.85 (NS) \\
\hline \multicolumn{7}{|c|}{ Macroscopic tumour size ${ }^{f}$} \\
\hline $\begin{array}{l}\leqslant 25 \mathrm{~mm} \\
>25 \mathrm{~mm}\end{array}$ & $\begin{array}{l}222(49.8) \\
224(50.2)\end{array}$ & $\begin{array}{r}62(37.3) \\
104(62.7)\end{array}$ & 0.000017 & $\begin{array}{l}11(33.3) \\
22(66.7)\end{array}$ & $\begin{array}{l}211(51.1) \\
202(48.9)\end{array}$ & 0.050 \\
\hline \multicolumn{7}{|l|}{$E R \alpha$ status } \\
\hline $\begin{array}{l}\text { Negative } \\
\text { Positive }\end{array}$ & $\begin{array}{l}116(25.6) \\
338(74.4)\end{array}$ & $\begin{array}{r}48(28.7) \\
119(71.3)\end{array}$ & 0.021 & $\begin{array}{l}11(33.3) \\
22(66.7)\end{array}$ & $\begin{array}{l}105(24.9) \\
316(75.1)\end{array}$ & 0.29 (NS) \\
\hline \multicolumn{7}{|l|}{ PR status } \\
\hline $\begin{array}{l}\text { Negative } \\
\text { Positive }\end{array}$ & $\begin{array}{l}192(42.3) \\
262(57.7)\end{array}$ & $\begin{array}{l}83(49.7) \\
84(50.3)\end{array}$ & 0.0024 & $\begin{array}{l}19(57.6) \\
14(42.4)\end{array}$ & $\begin{array}{l}173(41.1) \\
248(58.9)\end{array}$ & 0.065 (NS) \\
\hline \multicolumn{7}{|l|}{ ERBB2 status } \\
\hline $\begin{array}{l}\text { Negative } \\
\text { Positive }\end{array}$ & $\begin{array}{r}357(78.6) \\
97(21.4)\end{array}$ & $\begin{array}{r}127(76.0) \\
40(24.0)\end{array}$ & 0.17 (NS) & $\begin{array}{r}28(84.8) \\
5(15.2)\end{array}$ & $\begin{array}{r}329(78.1) \\
92(21.9)\end{array}$ & 0.37 (NS) \\
\hline \multicolumn{7}{|c|}{ Molecular subtypes } \\
\hline $\begin{array}{l}\mathrm{HR}-\text { ERBB2- } \\
\mathrm{HR}-\text { ERBB2 } \\
\mathrm{HR}+\text { ERBB2- } \\
\mathrm{HR}+\text { ERBB2 + }\end{array}$ & $\begin{array}{c}68(15.0) \\
43(9.5) \\
289(63.7) \\
54(11.9) \\
\end{array}$ & $\begin{array}{r}26(15.6) \\
21(12.6) \\
101(60.5) \\
19(11.4) \\
\end{array}$ & 0.032 & $\begin{aligned} & 8(24.2) \\
& 3(9.1) \\
& 20(60.6) \\
& 2(6.1) \\
&\end{aligned}$ & $\begin{aligned} 60(14.3) \\
40(9.5) \\
269(63.9) \\
52(12.4) \\
\end{aligned}$ & 0.38 (NS) \\
\hline \multicolumn{7}{|c|}{$\begin{array}{l}\text { a Abbreviations: NS = not significant; SBR=Scar } \\
\text { b } \chi^{2} \text { test. } \\
\text { c }_{\text {SBR classification. }} \\
d_{\text {Information available for } 445 \text { patients. }} \\
e_{\text {Information available for } 453 \text { patients. }} \\
\mathbf{f}_{\text {Information available for } 446 \text { patients. }}\end{array}$} \\
\hline
\end{tabular}

$64.9 \%$ at 10 years (120 months). In contrast, MFS was not affected by Gadkin overexpression in a statistically significant manner $(P=0.14)$. So among the Arp $2 / 3$ inhibitory proteins analysed, only Arpin downregulation is associated with a poor prognosis in breast cancer.

In an attempt to generalise these results to another cancer, we examined a cohort of patients affected with colorectal cancer. Even though this cohort was too recent to perform survival analysis, we found that the expression of Arpin was downregulated during progression of colorectal cancer. Indeed, adenomas displayed a significant downregulation compared with normal colon, whereas adenocarcinomas represented a further downregulation compared with adenomas (Supplementary Figure 1). Metastasis in the liver displayed a similar downregulation as primary adenocarcinomas. In conclusion, Arpin downregulation at the mRNA level is observed in both colorectal and breast cancer and this downregulation is significantly associated with high grade. We further characterised Arpin downregulation in breast cancer, because of its observed association with poor prognosis.

Analysis of Arpin expression at the protein level. We next sought to verify that Arpin downregulation at the mRNA level was accompanied by downregulation at the protein level. We first used
RPPA, where protein extracts from tumours are printed on microscope slides, which are then incubated with validated highquality antibodies and labelled with fluorescent secondary antibodies. Custom-made Arpin antibodies were produced against full-length Arpin protein and affinity purified. They give a major band at the right size by western blot analysis in several tumour extracts (Supplementary Figure 2). This band is at the same size as the one detected in cells in culture, and this band disappears upon RNAi-mediated depletion (Dang et al, 2013).

Out of the initial 454 patients, 233 biopsy samples were available to produce RPPA microscope slides. RPPA and qRT-PCR analyses on the same samples allowed us to examine the correlation between Arpin protein levels and mRNA levels. A significant correlation was found with Spearman correlation coefficient of $+0.42(P=0.0000008)$, indicating that the major downregulation observed in tumours is exerted at the mRNA level and that this mRNA regulation impacts Arpin expression at the protein level.

In breast biopsies, a variable amount of healthy tissue is removed and even within tumours, carcinoma cells are surrounded by healthy cells and stroma. Therefore, we next sought to examine Arpin expression at the cellular level by immunofluorescence. We studied cryosections of breast tumours after immunofluorescence with the same characterised affinity purified antibodies targeting 
full-length Arpin. Again these antibodies were validated, as they were shown to label cells expressing Arpin, but not the ones depleted of Arpin through the use of siRNA (Dang et al, 2013). One section was stained with Arpin antibodies and a consecutive one by a combination of monoclonal antibodies targeting either keratin $8(\mathrm{~K} 8)$, which is expressed in luminal epithelial cells, or keratin 17 (K17), which is expressed in surrounding myoepithelial cells of mammary ducts (Guelstein et al, 1988).

In normal tissue adjacent to tumours, we found strong Arpin expression in cells that form mammary gland acini and much weaker expression in stromal cells surrounding acini (Figure 2). Both K8- and K17-expressing cells were brightly stained for Arpin. In mammary carcinomas, however, Arpin expression was reduced in line with the mRNA and protein downregulation observed by qRT-PCR and RPPA in the retrospective cohort. In situ carcinomas were characterised by an expansion of the K8-positive population that filled lumen of acini. Carcinoma cells were poorly stained with Arpin antibodies, in contrast with surrounding K17expressing myoepithelial cells, which remained positive. In the case of invasive carcinomas, tumour cells stained by the K8 marker were not stained by Arpin antibodies.

To evaluate whether this downregulation was characteristic of carcinoma transformation or was simply related to cell proliferation, we observed a few biopsies of fibrocystic diseases, where proliferation is benign. No Arpin downregulation was observed in these cases (Supplementary Figure 3), further indicating that Arpin downregulation is associated with cancer progression. We also examined axillary lymph nodes for metastasis. Lymphocytes in lymph nodes were stained by Arpin antibodies. However, when metastases were detected in lymph nodes, these K8-positive tumour cells were also poorly stained with Arpin antibodies, like carcinoma cells of primary tumours (Figure 3).

In the series of breast tumours examined, we quantified immunofluorescence intensity from multiple fields of view, and found that indeed in most cases, in situ, invasive and metastatic carcinoma cells significantly loose Arpin expression compared with normal epithelial cells of mammary acini (Supplementary Figure 4).

Arpin expression and its role in breast cell lines. Numerous transformed and untransformed cell lines have been established from mammary carcinomas or breast tissues. We prepared mRNA and protein extracts from 32 of these breast cell lines, and assayed Arpin expression. Nine out of thirty-two cell lines displayed undetectable level of Arpin by western blot analysis (Supplementary Figure 5A). qRT-PCR is a more sensitive technique and Arpin was detected in all cell lines. A significant correlation was found between Arpin mRNA and protein levels in breast cell lines (Spearman coefficient of $+0.45, P=0.009$ ). This result suggests that Arpin downregulation in breast cell lines is primarily exerted at the transcriptional level, as in mammary tumours.

The diversity of these cell lines reflecting different breast tumour origins and different levels of invasiveness allowed us to examine potential correlations between Arpin expression and these parameters. Loss of Arpin expression was not associated with increased invasiveness of these cell lines. For example, the untransformed hTERT-HME1 cells had lost Arpin expression, as well as the weakly invasive MCF7 cells, whereas the classical invasive MDA-MB-231 cells had a detectable expression level. Similarly, we did not detect any obvious correlation with the origin of the breast cell lines. Loss of Arpin expression was observed in lines from all origins, normal-like, basal, luminal and Her2-positive cancer (Supplementary Figure 5B).

To better understand the role of Arpin in breast tumour cell lines, we thus depleted Arpin from an Arpin-expressing breast cancer cell line. We used clones of MDA-MB-231 stably depleted of Arpin through the expression of shRNAs (Figure 4A, Dang et al, 2013). In line with an inhibitory role of Arpin in cell migration, we found that Arpin-depleted cells were faster than the control cells to

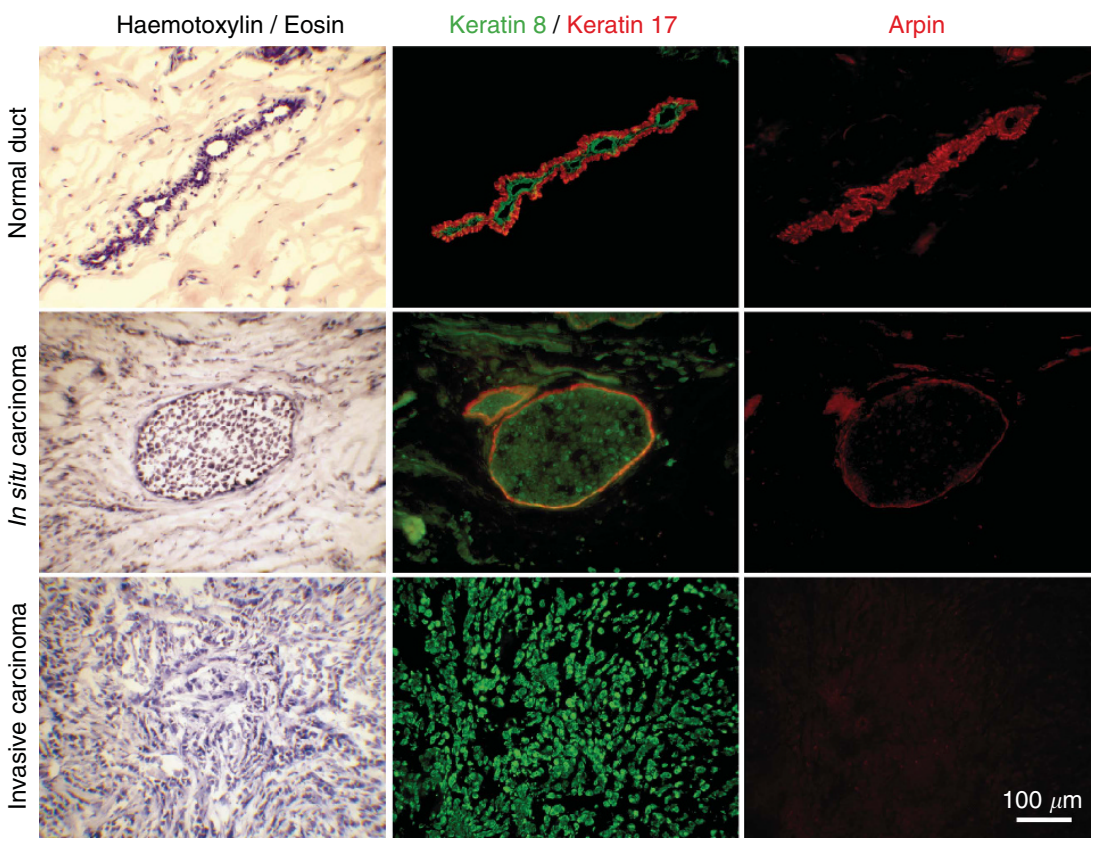

Figure 2. Loss of Arpin expression in breast carcinoma cells. Arpin expression was examined by immunofluorescence in consecutive cryosections of breast biopsies. Normal alveolar ducts are composed of two epithelial layers; the inner luminal layer expresses keratin 8 , whereas the surrounding basal myoepithelial layer expresses keratin 17. Arpin is highly expressed in both epithelial cell types compared with fibroblasts in the conjunctive tissue. Breast carcinomas in situ display proliferation of luminal epithelial cells, where Arpin expression is reduced. Arpin expression is retained in the myoepithelial layer. Invasive cells, having lost all epithelial characteristics, even though they still express keratin 8 , displayed strongly suppressed Arpin expression. 


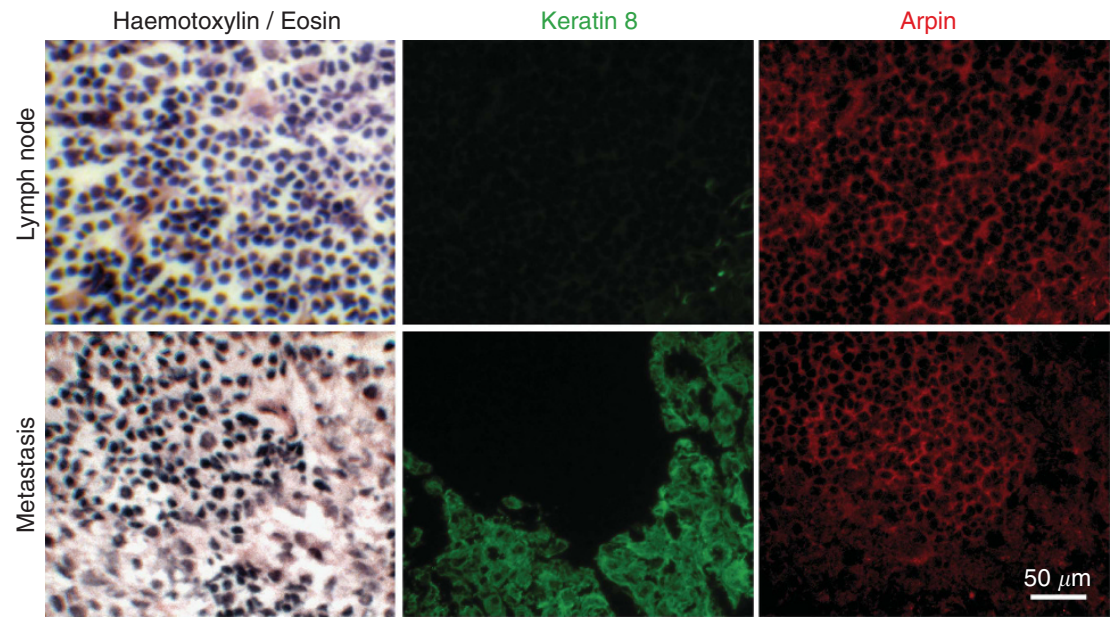

Figure 3. Loss of Arpin expression in metastatic cells in lymph node. Metastatic carcinoma cells settled in a regional lymph node displayed reduced Arpin expression compared with the surrounding lymphocytes.

close a wound (Figure 4C and Supplementary Movie 1). In addition to a role in migration, we found here that Arpin controls cell proliferation. Indeed, growth curves indicated that Arpin depletion increases cell proliferation (Figure 4B). Arpin-depleted MDA-MB-231 clones were found to generate more transformation foci than controls (Figure 4D). When anchorage-independent growth was tested in the classical soft agar assay, Arpin-depleted clones formed larger colonies than control cells (Figure 4E). Altogether, these assays suggest that loss of Arpin expression is associated with enhanced growth and migration of tumour cells, in line with the poor prognosis of breast cancer patients.

Arpin antagonism with the WAVE complex. As Arpin antagonises the WAVE complex, the Arp2/3-activating molecular machine that drives cell migration (Dang et al, 2013), we decided to examine expression of the genes encoding WAVE complex subunits in the cohort of breast cancer patients. The WAVE complex is a multiprotein complex composed of five canonical subunits, CYFIP, NCKAP, WAVE, ABI and BRK1 (Derivery and Gautreau, 2010). The CYFIP and NCKAP families of subunits are encoded by two paralogous genes each. The WAVE and ABI families of subunits are encoded by three paralogous genes each. Only the BRK1 subunit is encoded by a single gene in the human genome. Out of these 11 genes, we identified NCKAP1 as the gene, whose variations of expression were the most strongly associated with a poor prognosis for patients.

NCKAP1 expression was associated with hormone receptor status, ERBB2 status and with the molecular subtype of breast cancers (Supplementary Table 3). The group displaying high NCKAP1 expression was associated with reduced MFS with a significant $P$ of 0.0037 (Figure 5A). In patients displaying high NCKAP 1 expression, there was only $70.0 \%$ MFS $v s 77.5 \%$ at 5 years (60 months) and $57.6 \%$ vs $68.7 \%$ at 10 years (120 months). As the WAVE complex activates the Arp $2 / 3$ complex and Arpin inactivates it, it appeared logical that the same poor outcome could be produced by NCKAP1 upregulation or Arpin downregulation. We thus combined Arpin low and/or NCKAP1 high in the same category (Supplementary Table 4) and plotted MFS. This combination significantly increased the prognostic power of these genetic alterations. Arpin low and/or NKCAP1 high was associated with a much reduced MFS with a very significant $P$ of 0.000064 . In patients displaying Arpin low and/or NKCAP1 high, there was only $67.2 \%$ MFS vs $80.6 \%$ at 5 years (60 months) and $55.1 \%$ vs $72.1 \%$ at 10 years (120 months). Multivariate analysis using a Cox proportional hazard model assessed the predictive value for MFS of the significant parameters on univariate analysis, that is, tumour size, SBR histological grade, lymph node status, hormone receptor status and 'Arpin low and/or NKCAP1 high'. The prognostic significance of tumour size $(P=0.00047)$, SBR histological grade $(P=0.049)$ and the 'Arpin low and/or NKCAP1 high' category $(P=0.0012)$ persisted (Figure $5 \mathrm{C})$, indicating that the latter parameter is a new independent prognostic factor of MFS. The vast majority of tumours in this category displayed either Arpin low or NCKAP1 high (Figure 5D), highlighting further that downregulating the inhibitor or up-regulating the activator are two mutually exclusive ways to produce the same outcome.

\section{DISCUSSION}

In this study, we examined three Arp2/3 inhibitory proteins for their potential deregulation in breast cancer. Whereas the variations of Gadkin and PICK1 did not have prognostic value, we found that Arpin downregulation at the mRNA level was associated with poor prognosis in a cohort of 446 patients affected with breast cancer. Downregulation of Arpin mRNA was observed in both breast and colorectal cancers. In both cases, this downregulation was more pronounced in advanced stages. Using RPPA and immunofluorescence of biopsies, Arpin downregulation was also observed at the protein level in breast carcinomas. A number of breast cancer lines in culture display a similar Arpin downregulation.

Arpin is a conserved Arp $2 / 3$ inhibitory protein that renders membrane protrusions unstable and which, as a consequence, inhibits cell migration and invasion in 3D collagen gels (Dang et al, 2013; Gorelik and Gautreau, 2015). In this context, pronounced downregulation of Arpin in advanced stages of cancers therefore seems to make sense with respect to the reduced MFS. However, Arpin downregulation is also observed by immunofluorescence of patient tumours in in situ carcinomas, and not only when carcinomas are invasive. By depleting Arpin from MDA-MB-231 cells using stable shRNA expression, we found here that Arpin not only inhibits cell migration in a wound healing assay, but also restricts proliferation and anchorage-independent growth. This newly reported role of Arpin is consistent with the idea that it is advantageous for tumour cells to lose Arpin expression as early as the in situ stage. 
A
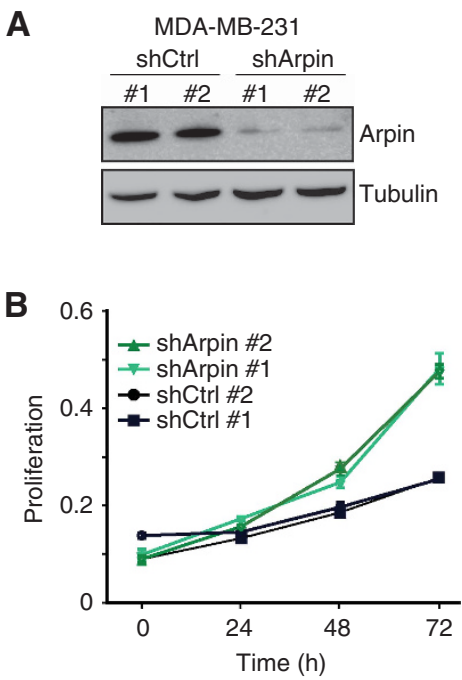

D

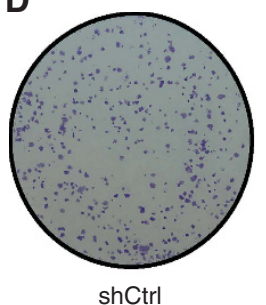

$\mathbf{E}$

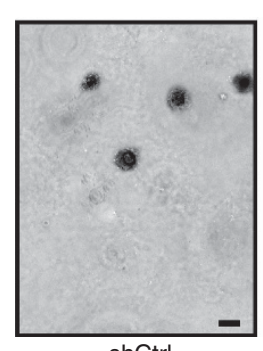

shCtrl
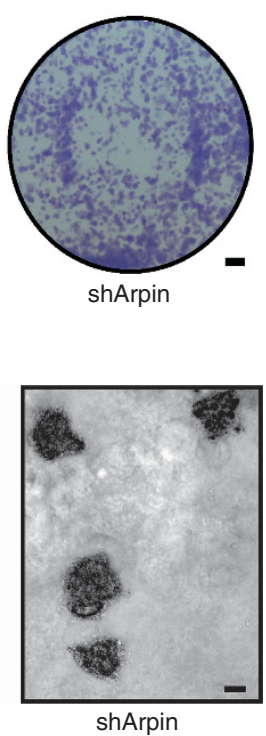

C
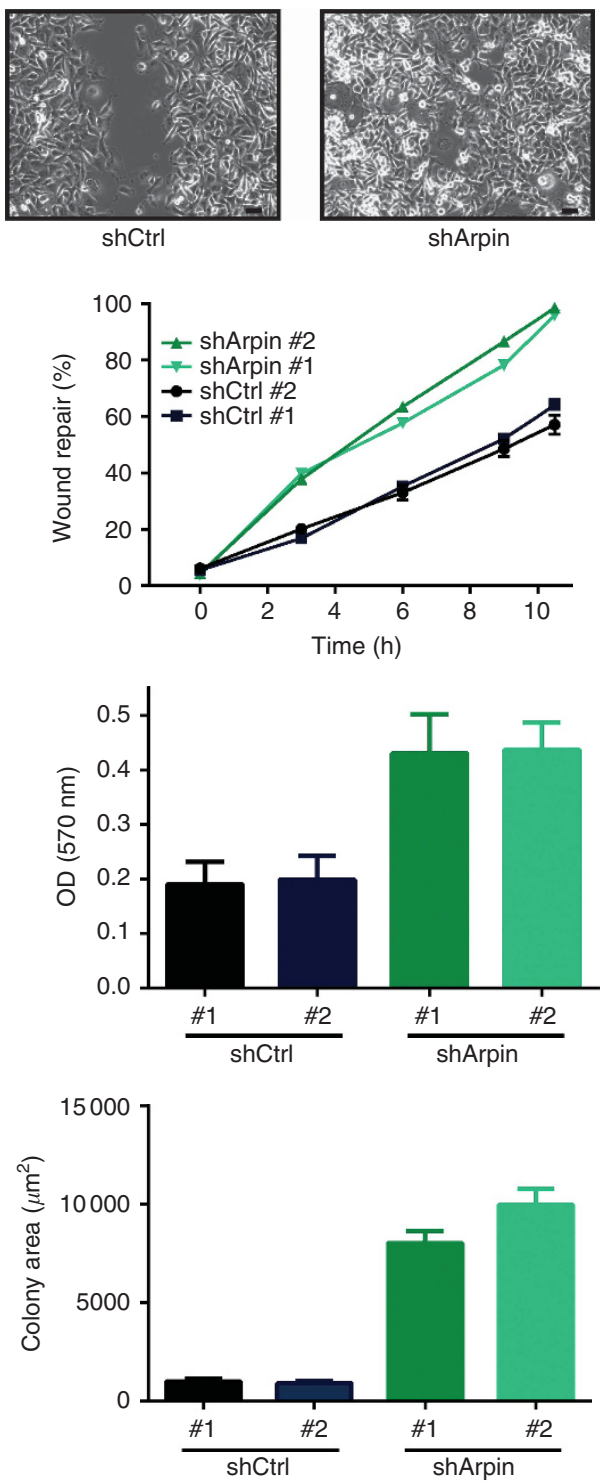

Figure 4. Arpin-depleted MDA-MB-231 cells display increased migration and proliferation. (A) Two MDA-MB-231 clones stably depleted of Arpin and two clones transfected with non-targeting shRNA (shCtrl) were analysed by western blot for their Arpin content. (B) MDA-MB-231 clones stably depleted of Arpin close a wound faster than controls. Pictures were taken after $10 \mathrm{~h}$ of wound healing. Bar: $20 \mu \mathrm{m}$. (C) Growth curves of the clones. MDA-MB-231 clones stably depleted of Arpin proliferate faster than controls. (D) Transformation foci stained with crystal violet and quantification of the stain. Mean and s.e.m. are represented. Bar: 2 mm. (E) Anchorage-independent growth in soft agar. MDA-MB-231 clones stably depleted of Arpin generate larger colonies than controls. Bar: $20 \mu \mathrm{m}$. Mean and s.e.m. are represented.

We previously found that Arpin antagonises the WAVE complex (Dang et al, 2013). The WAVE complex activates the Arp2/3 complex at the leading edge of migrating cells, whereas Arpin inactivates it at the same location. Importantly, both Arp2/3 regulators are under the control of the Rac small GTPase (Dang et al, 2013). Rac is well known to control membrane protrusions and cell migration (Insall and Machesky, 2009). However, Rac, like Arpin, is also critical for the proliferation of breast cancer cells (Yang et al, 2008; Arias-Romero et al, 2010; Ebi et al, 2013). The antagonism between Arpin and the WAVE complex prompted us to examine the expression of the WAVE complex subunits in the same cohort of breast cancer patients. As reminded in the introduction, altered expression of WAVE complex subunits had previously been associated with the progression of a variety of cancers, but with up- or downregulations depending on the specific paralogous subunit examined. In our cohort of breast cancer patients, we found a similar complexity in opposite deregulations of different subunits. We would like in the future to relate these observations to the molecular physiology of the different WAVE complexes. However, despite this complexity, altered expression of the NCKAP1 subunit stood alone, as being the most strongly associated with prognosis of breast cancer patients. This result was not reported before in any cancer, most likely, because to our knowledge, WAVE complex subunits had never been examined systematically.

Strikingly consistent with molecular studies showing that Arpin inhibits the Arp $2 / 3$ complex and that the WAVE complex activates it (Derivery and Gautreau, 2010; Dang et al, 2013), we found that low Arpin levels or high NCKAP1 levels are similarly associated with poor prognosis in breast cancer patients. These results are also consistent with the many studies reporting that Arp $2 / 3$ overexpression is associated with poor prognosis in various cancers (Otsubo et al, 2004; Semba et al, 2006; Iwaya et al, 2007a, b; Laurila et al, 2009). Combining tumours displaying low Arpin and/or high 

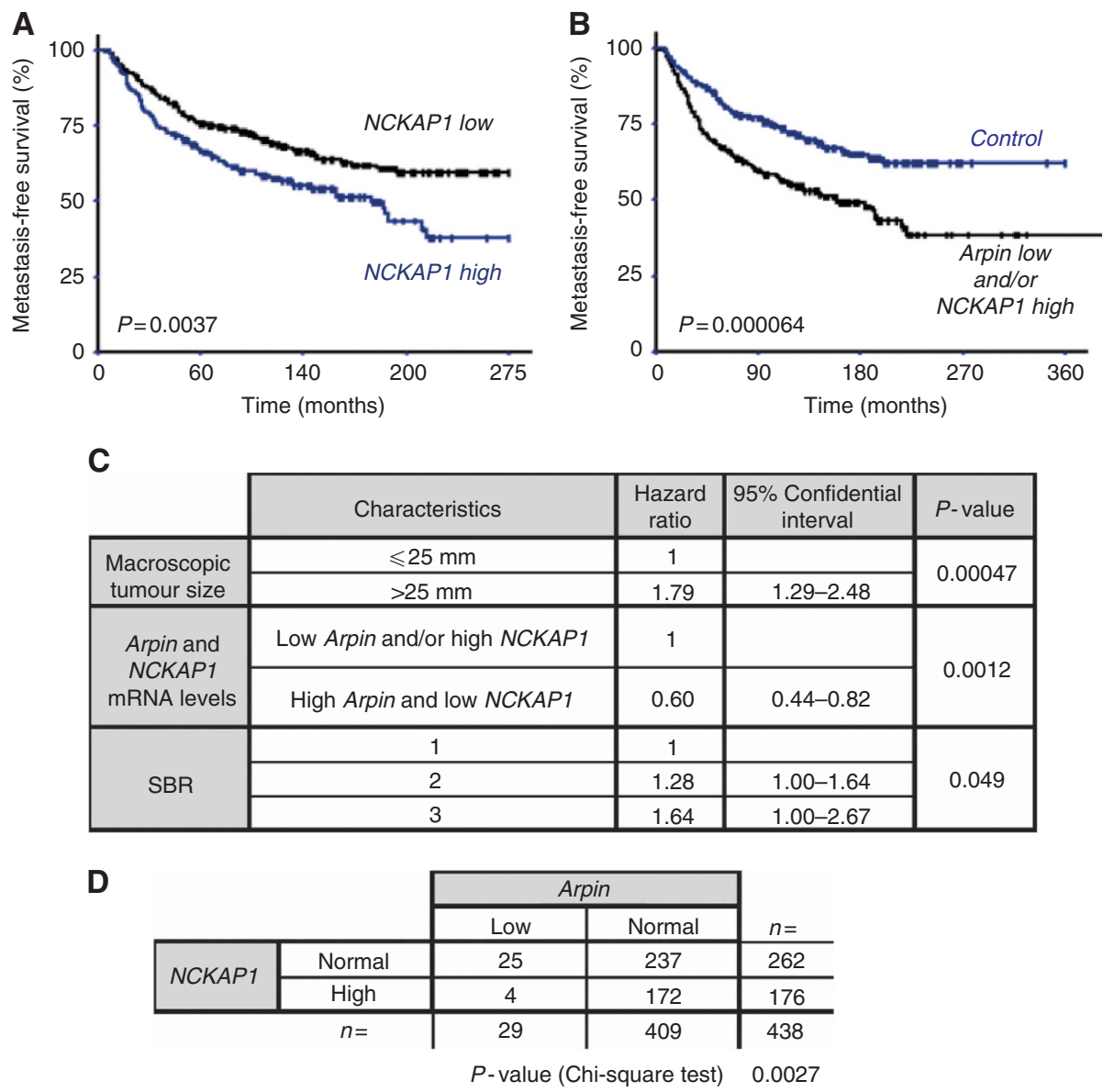

Figure 5. Patients displaying Arpin low or NCKAP1 high have similar poor outcomes in breast cancer (A) Patients exhibiting high NCKAP1 have reduced MFS (log-rank test using optimal cutoff of 1.34; $P=0.0037$ ). (B) Patients exhibiting low Arpin and/or high NCKAP1 have further reduced MFS (log-rank test; $P=0.000064)$. (C) Cox multivariate analysis of MFS shows that low Arpin and/or high NCKAP1 is an independent prognosis factor of MFS $(P=0.0012)$. (D) $\chi^{2}$ test indicates a significant mutual exclusion between Arpin low and NCKAP1 high groups $(P=0.0027)$.

NCKAP1 significantly improved the association with poor prognosis and this criterium of 'low Arpin and/or high NCKAP1' was found to be an independent prognostic factor in multivariate analysis. In our cohort, high NCKAP1 represented $\sim 40 \%$ of the cases, and low Arpin 7\%. High NCKAP1 and low Arpin appeared mutually exclusive, as $<1 \%$ of tumours displayed both deregulations. Alterations in this Arp $2 / 3$ regulatory circuit thus already represent almost half of the breast cancer cases, just taking into account the NCKAP1 subunit. This number can further increase when WAVE complex subunits will be studied in combinations. This development will require thorough statistical analyses and a molecular dissection of the combinatorial complexity of WAVE complexes.

In conclusion, our data reveal that the pattern of expression of genes encoding Arpin and WAVE complex subunits should be taken into account for a refined prognosis of breast cancer and might also indicate patients, which would potentially benefit from treatments with available drugs that inhibit the Arp $2 / 3$ complex.

\section{ACKNOWLEDGEMENTS}

We thank Caroline Lecerf and Céline Baldeyron for performing RPPA experiments, Vera Dugina for advice on immunofluorescence of biopsies, Virginie Maire for providing protein extracts from the breast cell lines, Nadia Kurochkina for help with western blots and Dominique Lallemand for critically reading the manuscript. This work was supported by ANR-11-BSV8-0010-02 to A.G., Institut National du Cancer (INCA_6521) to A.G. and I.B., Fondation ARC pour la Recherche sur le Cancer (PGA120140200831) to A.G. and I.B., the Comité départemental des Hauts-de-Seine de la Ligue Nationale Contre le Cancer to I.B., an educational fellowship SP-6587.2013.4 to M.E.L. and RFBR grant 14-04-91056CNRC_a to A.Y.A. The RPPA platform is supported by Cancéropôle Ile-de-France.

\section{CONFLICT OF INTEREST}

S.V., A.Y.A. and A.G. submitted a patent with the part of the current results showing that Arpin is frequently downregulated in breast cancer and that Arpin downregulation is associated with poor prognostic for patients. The patent was accepted by the European Patent Office (Patent EP 2842566 A1 priority 03/09/ 2013, published: 04/05/2015).

\section{REFERENCES}

Arias-Romero LE, Villamar-Cruz O, Pacheco A, Kosoff R, Huang M, Muthuswamy SK, Chernoff J (2010) A Rac-Pak signaling pathway is essential for ErbB2-mediated transformation of human breast epithelial cancer cells. Oncogene 29: 5839-5849. 
Bieche I, Parfait B, Laurendeau I, Girault I, Vidaud M, Lidereau R (2001) Quantification of estrogen receptor alpha and beta expression in sporadic breast cancer. Oncogene 20: 8109-8115.

Dang I, Gorelik R, Sousa-Blin C, Derivery E, Guérin C, Linkner J, Nemethova M, Dumortier JG, Giger FA, Chipysheva TA, Ermilova VD, Vacher S, Campanacci V, Herrada I, Planson A-G, Fetics S, Henriot V, David V, Oguievetskaia K, Lakisic G, Pierre F, Steffen A, Boyreau A, Peyriéras N, Rottner K, Zinn-Justin S, Cherfils J, Bièche I, Alexandrova AY, David NB, Small JV, Faix J, Blanchoin L, Gautreau A (2013) Inhibitory signalling to the Arp2/3 complex steers cell migration. Nature 503: 281-284.

Derivery E, Gautreau A (2010) Generation of branched actin networks: assembly and regulation of the N-WASP and WAVE molecular machines. Bioessays 32: 119-131.

Ebi H, Costa C, Faber AC, Nishtala M, Kotani H, Juric D, Pelle Della P, Song Y, Yano S, Mino-Kenudson M, Benes CH, Engelman JA (2013) PI3K regulates MEK/ERK signaling in breast cancer via the Rac-GEF, P-Rex1. Proc Natl Acad Sci USA 110: 21124-21129.

Frugtniet B, Jiang WG, Martin TA (2015) Role of the WASP and WAVE family proteins in breast cancer invasion and metastasis. Breast Cancer (Dove Med Press) 7: 99-109.

Gorelik R, Gautreau A (2015) The Arp2/3 inhibitory protein Arpin induces cell turning by pausing cell migration. Cytoskeleton 72: 362-371.

Guelstein VI, Tchypysheva TA, Ermilova VD, Litvinova LV, Troyanovsky SM, Bannikov GA (1988) Monoclonal antibody mapping of keratins 8 and 17 and of vimentin in normal human mammary gland, benign tumors, dysplasias and breast cancer. Int J Cancer 42: 147-153.

Insall RH, Machesky LM (2009) Actin dynamics at the leading edge: from simple machinery to complex networks. Dev Cell 17: 310-322.

Iwaya K, Norio K, Mukai K (2007a) Coexpression of Arp2 and WAVE2 predicts poor outcome in invasive breast carcinoma. Mod Pathol 20: 339-343.

Iwaya K, Oikawa K, Semba S, Tsuchiya B, Mukai Y, Otsubo T, Nagao T, Izumi M, Kuroda M, Domoto H, Mukai K (2007b) Correlation between liver metastasis of the colocalization of actin-related protein 2 and 3 complex and WAVE2 in colorectal carcinoma. Cancer Sci 98: 992-999.

Krause M, Gautreau A (2014) Steering cell migration: lamellipodium dynamics and the regulation of directional persistence. Nat Rev Mol Cell Biol 15: 577-590.

Kulkarni S, Augoff K, Rivera L, McCue B, Khoury T, Groman A, Zhang L, Tian L, Sossey-Alaoui K (2012) Increased expression levels of WAVE3 are associated with the progression and metastasis of triple negative breast cancer. PLoS ONE 7: e42895.

Kurisu S, Takenawa T (2010) WASP and WAVE family proteins: friends or foes in cancer invasion? Cancer Sci 101: 2093-2104.

Laurila E, Savinainen K, Kuuselo R, Karhu R, Kallioniemi A (2009) Characterization of the 7q21-q22 amplicon identifies ARPC1A, a subunit of the Arp2/3 complex, as a regulator of cell migration and invasion in pancreatic cancer. Genes Chromosom Cancer 48: 330-339.

Liu Z, Yang X, Chen C, Liu B, Ren B, Wang L, Zhao K, Yu S, Ming H (2013) Expression of the Arp2/3 complex in human gliomas and its role in the migration and invasion of glioma cells. Oncol Rep 30: 2127-2136.

Maire V, Baldeyron C, Richardson M, Tesson B, Vincent-Salomon A, Gravier E, Marty-Prouvost B, De Koning L, Rigaill G, Dumont A, Gentien D, Barillot E, Roman-Roman S, Depil S, Cruzalegui F, Pierré A, Tucker GC, Dubois T (2013) TTK/hMPS1 is an attractive therapeutic target for triplenegative breast cancer. PLOS ONE 8: e63712.

Maritzen T, Zech T, Schmidt MR, Krause E, Machesky LM, Haucke V (2012) Gadkin negatively regulates cell spreading and motility via sequestration of the actin-nucleating ARP2/3 complex. Proc Natl Acad Sci U S A 109: 10382-10387.

Martin TA, Pereira G, Watkins G, Mansel RE, Jiang WG (2007) N-WASP is a putative tumour suppressor in breast cancer cells, in vitro and in vivo, and is associated with clinical outcome in patients with breast cancer. Clin Exp Metastasis 25: 97-108.

Otsubo T, Iwaya K, Mukai Y, Mizokami Y, Serizawa H, Matsuoka T, Mukai K (2004) Involvement of Arp2/3 complex in the process of colorectal carcinogenesis. Mod Pathol 17: 461-467.

Rauhala HE, Teppo S, Niemelä S, Kallioniemi A (2013) Silencing of the ARP2/ 3 complex disturbs pancreatic cancer cell migration. Anticancer Res 33: $45-52$.

Rocca DL, Martin S, Jenkins EL, Hanley JG (2008) Inhibition of Arp2/3mediated actin polymerization by PICK1 regulates neuronal morphology and AMPA receptor endocytosis. Nat Cell Biol 10: 259-271.

Rondeau S, Vacher S, De Koning L, Briaux A, Schnitzler A, Chemlali W, Callens C, Lidereau R, Bieche I (2015) ATM has a major role in the double-strand break repair pathway dysregulation in sporadic breast carcinomas and is an independent prognostic marker at both mRNA and protein levels. Br J Cancer 112: 1059-1066.

Rotty JD, Wu C, Bear JE (2013) New insights into the regulation and cellular functions of the ARP2/3 complex. Nat Rev Mol Cell Biol 14: 7-12.

Semba S, Iwaya K, Matsubayashi J, Serizawa H, Kataba H, Hirano T, Kato H, Matsuoka T, Mukai K (2006) Coexpression of actin-related protein 2 and Wiskott-Aldrich syndrome family verproline-homologous protein 2 in adenocarcinoma of the lung. Clin Cancer Res 12: 2449-2454.

Silva JM, Ezhkova E, Silva J, Heart S, Castillo M, Campos Y, Castro V, Bonilla F, Cordon-Cardo C, Muthuswamy SK, Powers S, Fuchs E, Hannon GJ (2009) Cyfip1 is a putative invasion suppressor in epithelial cancers. Cell 137: 1047-1061.

Sossey-Alaoui K, Safina A, Li X, Vaughan MM, Hicks DG, Bakin AV, Cowell JK (2007) Down-regulation of WAVE3, a metastasis promoter gene, inhibits invasion and metastasis of breast cancer cells. Am J Pathol 170: $2112-2121$.

Sowalsky AG, Sager R, Schaefer RJ, Bratslavsky G, Pandolfi PP, Balk SP, Kotula L (2015) Loss of Wave1 gene defines a subtype of lethal prostate cancer. Oncotarget 6: 12383-12391.

Tang H, Li A, Bi J, Veltman DM, Zech T, Spence HJ, Yu X, Timpson P, Insall RH, Frame MC, Machesky LM (2013) Loss of Scar/WAVE complex promotes N-WASP- and FAK-dependent invasion. Curr Biol 23: 107-117.

Xiong X, Chorzalska A, Dubielecka PM, White JR, Vedvyas Y, Hedvat CV, Haimovitz-Friedman A, Koutcher JA, Reimand J, Bader GD, Sawicki JA, Kotula L (2012) Disruption of Abi1/Hssh3bp1 expression induces prostatic intraepithelial neoplasia in the conditional Abi1/Hssh3bp1 KO mice. Oncogenesis 1: e26.

Yamaguchi H, Lorenz M, Kempiak S, Sarmiento C, Coniglio S, Symons M, Segall J, Eddy R, Miki H, Takenawa T, Condeelis J (2005) Molecular mechanisms of invadopodium formation: the role of the N-WASP-Arp2/3 complex pathway and cofilin. J Cell Biol 168: 441-452.

Yang C, Klein EA, Assoian RK, Kazanietz MG (2008) Heregulin beta1 promotes breast cancer cell proliferation through Rac/ERK-dependent induction of cyclin D1 and p21Cip1. Biochem J 410: 167-175.

This work is published under the standard license to publish agreement. After 12 months the work will become freely available and the license terms will switch to a Creative Commons AttributionNonCommercial-Share Alike 4.0 Unported License. 